\title{
Analysis of multiple quaternary ammonium compounds in the brain using tandem capillary column separation and high resolution mass spectrometric detection
}

\author{
Sara Falasca ${ }^{a}$, Filomena Petruzziello ${ }^{a}$, Robert Kretz ${ }^{a}$, Gregor Rainer ${ }^{\mathrm{a}, \mathrm{b}}$, Xiaozhe Zhang a,* \\ a Visual Cognition Laboratory, Department of Medicine, Chemin du Musee 5, University of Fribourg, Fribourg CH-1700, Switzerland \\ ${ }^{\mathrm{b}}$ Fribourg Center for Cognition, University of Fribourg, Fribourg CH-1700, Switzerland
}

\begin{abstract}
Endogenous quaternary ammonium compounds are involved in various physiological processes in the central nervous system. In the present study, eleven quaternary ammonium compounds, including acetylcholine, choline, carnitine, acetylcarnitine and seven other acylcarnitines of low polarity, were analyzed from brain extracts using a two dimension capillary liquid chromatography-Fourier transform mass spectrometry method. To deal with their large difference in hydrophobicities, tandem coupling between reversed phase and hydrophilic interaction chromatography columns was used to separate all the targeted quaternary ammonium compounds. Using high accuracy mass spectrometry in selected ion monitoring mode, all the compounds could be detected from each brain sample with high selectivity. The developed method was applied for the relative quantification of these quaternary ammonium compounds in three different brain regions of tree shrews: prefrontal cortex, striatum, and hippocampus. The comparative analysis showed that quaternary ammonium compounds were differentially distributed across the three brain areas. The analytical method proved to be highly sensitive and reliable for simultaneous determination of all the targeted analytes from brain samples.
\end{abstract}

\section{Introduction}

Endogenous quaternary ammonium compounds (QACs) are broadly distributed in various tissues including the central nervous system, and many QACs have important biochemical functions in brain $[1,2]$. For example, acetylcholine is a critical neuromodulator that plays a key role in diverse cognitive functions [3,4]. Choline, the product/precursor of acetylcholine, is an important metabolite [5] and can also act as a nicotinic receptor agonist [6]. The acylcarnitines are a class of QACs that are derivates of carnitine [7]. Structurally, all carnitines, acetylcholine and choline share a trimethyl quaternary ammonium group, and in particular, acetylcarnitine bears very close structural resemblance to acetylcholine (see their structures in Fig. 1). There are also a number of documented functional links between carnitines and acetylcholine in brain [1]. For example, acetylcarnitine can act as a source of acetyl moieties and thus provide a precursor for the production of acetylcholine from choline [8-11]. In light of these interesting structural and physiological links, our study targets developing a mass spectrometry (MS)-based relative quantitative method that

\footnotetext{
* Corresponding author. Tel.: +41 263008910; fax: +41 263009734. E-mail address: xiaozhe.zhang@unifr.ch (X. Zhang).
}

enables sensitive monitoring of acetylcholine, choline, free carnitine, acetylcarnitine and seven acylcarnitines of low polarity from brain samples. We apply this method to brain samples obtained from tree shrews (Tupaia belangeri), a small mammalian species that is a close relative of primates including humans.

MS is a powerful method for the detection of QACs in electrospray mode (ESI) since QACs contain a stably charged quaternary ammonium group and thus exhibit high ionization efficiency [12]. To deal with the complexity of the biological samples, chromatographic separations are required in most cases to obtain high detection capability and high reproducibility in the analysis of QACs. Until now, there is still no analytical method available that allows the separation and detection of the targeted QACs in a single LC-MS analysis. With different side chains, these QACs form a class of molecules with different hydrophobicities, even though they share the structural identity of their ammonium groups. This hydrophobicity diversity poses challenges for the LC-MS analysis. Currently, the separation of QACs of high polarity, such as choline, acetylcholine, carnitine and acetylcarnitine, are commonly conducted using hydrophilic interaction chromatography (HILIC)-MS [13,14], ion-exchange chromatography [15,16], or ionpairing reversed phase (RP) chromatography [17-19]. To separate carnitines of both low and high polarities, pre-column derivatization has been used to improve their separation on common RP 


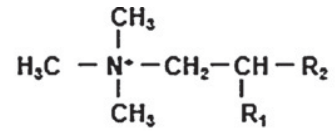

\begin{tabular}{|c|c|c|c|c|}
\hline Name & Abbrev. & $R_{i}$ & $R=$ & Mass \\
\hline Croline & $\mathrm{Ch}$ & $\mathrm{OH}$ & H & 104.1070 \\
\hline Acetyktroline & $\mathrm{ACh}$ & $\mathrm{OCOCH}_{3}$ & H & 146.1176 \\
\hline Carnitine & Cart & $\mathrm{OH}$ & $\mathrm{CH}_{2} \mathrm{COOH}$ & 162.1125 \\
\hline Acetylcarnitine & ACant & $\mathrm{OCOCH}_{3}$ & $\mathrm{CH}_{2} \mathrm{COOH}$ & 204.1230 \\
\hline Propionykcarnitine & Propiocant & $\mathrm{OCOCH}+\mathrm{CH}_{3}$ & $\mathrm{CH}_{2} \mathrm{COOH}$ & 218.1387 \\
\hline Hexaroykarnitine & HexCart & $\mathrm{OCO}\left(\mathrm{CH}_{3}\right)_{2} \mathrm{CH}_{3}$ & $\mathrm{CH}_{2} \mathrm{COOH}$ & 260.1856 \\
\hline Octanoykarnitine & OctCart & $\mathrm{OCO}\left(\mathrm{CH}_{2}\right)_{5} \mathrm{CH}_{3}$ & $\mathrm{CH}_{2} \mathrm{COOH}$ & 288.2169 \\
\hline Decaroykarnitine & DecaCart & $\mathrm{OCO}\left(\mathrm{CH}_{3} \mathrm{CH}_{3}\right.$ & $\mathrm{CH}_{2} \mathrm{COOH}$ & 316.2482 \\
\hline Lauroykarnitine & LauroCant & $\mathrm{OCO}\left(\mathrm{CH}_{2}\right)_{10} \mathrm{CH}_{3}$ & $\mathrm{CH}_{2} \mathrm{COOH}$ & 344.2795 \\
\hline Myristoykarnitine & MyrCart & $\mathrm{OCO}\left(\mathrm{CH}_{2}\right)_{2} \mathrm{CH}_{6}$ & $\mathrm{CH}_{2} \mathrm{COOH}$ & 372.3108 \\
\hline Palmitoykarniting & PalmCart & $\mathrm{OCO}\left(\mathrm{CH}_{2}\right)_{14} \mathrm{CH}_{3}$ & $\mathrm{CH}_{2} \mathrm{COOH}$ & 400.3421 \\
\hline
\end{tabular}

Fig. 1. Chemical structures of QACs analyzed and their accurate masses.

columns [20]. However, this method is only applicable to carnitines but not to acetylcholine or choline because these two QACs do not have carboxyl group available for such derivatization.

In the present study, we report a sensitive and reliable capillary liquid chromatography-Fourier transform mass spectrometry (LC-FT-MS) method that allows relative quantification of the targeted QACs from brain tissue. The method uses a simple, no-drying sample preparation that facilitates the extraction of all the targeted QACs from brain tissue. To deal with the analytical challenge caused by the diverse hydrophobicities of the QACs, tandem coupling of both RP and HILIC columns was used to separate all the targeted analytes in a single LC-MS analysis. The mobile phase delivered to MS was of high content acetonitrile and ion-pairing reagent free, and thus permitted high detection capability for the QACs. Our relative quantification method was proven to be of high selectivity, sensitivity and reproducibility, allowing profiling each QAC of interest in the different brain areas.

\section{Experimental}

\subsection{Reagents}

Choline $(\mathrm{Ch})$ chloride, acetylcholine (ACh) chloride, carnitine (Cart) hydrochloride and acetylcarnitine (ACart) hydrochloride were purchased from Sigma-Aldrich (St. Louis, MO, USA). Propionylcarnitine (PropioCart), hexanoylcarnitine (HexCart), octanoylcarnitine (OctCart), decanoylcarnitine (DecaCart), lauroylcarnitine (LauroCart), myristoylcarnitine (MyrCart) and palmitoylcarnitine (PalmCart) chloride were obtained from Tocris Bioscience (Ellisville, MO, USA). Choline-d9 chloride (Sigma-Aldrich), acetylcholine-d4 chloride (Medical Isotopes Inc., Pelham, NH, USA), carnitine-d3 hydrochloride, acetylcarnitine-d9 hydrochloride, octanoylcarnitine-d3 hydrochloride and palmitoylcarnitined3 hydrochloride (Cambridge Isotope Laboratories Inc., Andover, MA, USA) were used as internal standards (ISs). LC-MS grade formic acid, ammonium hydroxide solution, methanol and acetonitrile were supplied by Sigma-Aldrich. Water was obtained from a GenPure water system (TKA, Niederelbert, Germany).

\subsection{Preparation of standard and internal standard solutions}

Individual standard and internal standard stock solutions ( $1 \mathrm{mg} / \mathrm{mL}$ ) were prepared in $20 \%$ methanol containing $0.1 \%$ formic acid and stored at $-20^{\circ} \mathrm{C}$. These stock solutions were diluted to obtain a mixed standard and internal standard working solution in $25 \%$ acetonitrile containing $0.3 \%$ formic acid that was used to characterize the analytical performances of our tandem column HPLC-MS system (each analyte and IS at $50 \mathrm{nM}$ except for cholined9 at $1 \mu \mathrm{M}$ ). A mixed internal standard spiking solution in $20 \%$ acetonitrile containing $0.1 \%$ formic acid was also prepared from the stock solutions (acetylcholine-d4, carnitine-d3, acetylcarnitine-d9, octanoylcarnitine-d3, palmitoylcarnitine-d3 at $1 \mu \mathrm{M}$ and cholined9 at $20 \mu \mathrm{M})$.

\subsection{Animals and sample preparation}

Tree shrews (T. belangeri) were used in this study $(n=3)$. The animals were housed under constant temperature and humidity with free access to food and water. The handling of the animals and the experimental procedures were approved by the veterinary office of Fribourg, Switzerland. The tree shrews were sacrificed by decapitation after anesthetization with ketamine $(100 \mathrm{mg} / \mathrm{kg}$, Streuli Pharma AG, Uznach, Switzerland). The head was immediately heated up to $80^{\circ} \mathrm{C}$ in $16 \mathrm{~s}$ using microwave irradiation [21]. The brains were rapidly removed from the cranium and three areas (prefrontal cortex, striatum and hippocampus) were dissected. The tissues of each area were collected from three different animal brains and then pooled together, homogenized by the automated Precellys 24 homogenizer (Bertin Technologies, Montigny-le-Bretonneux, France) and divided in three samples that were processed separately. The striatum and hippocampus tissues (each $30 \mathrm{mg}$ ) were successively spiked with $20 \mu \mathrm{L}$ of the mixed internal standard spiking solution. The prefrontal cortex tissues (each $20 \mathrm{mg}$ ) were spiked with $13.3 \mu \mathrm{L}$ of the mixed internal standard spiking solution. The tissues were then homogenized in the Precellys 24 homogenizer, using ice-cold acetonitrile containing $0.3 \%$ formic acid $(100 \mu \mathrm{L}$ for striatum and hippocampus samples and $66.7 \mu \mathrm{L}$ for prefrontal cortex samples). The homogenates were centrifuged at $22,000 \times g$ for $20 \mathrm{~min}$ at $4^{\circ} \mathrm{C}$. The supernatants were collected and filtered by $0.20 \mu \mathrm{m}$ filter membranes (Millex-LG, Millipore, Billerica, MA, USA). The filtered supernatants were diluted four times with water containing $0.3 \%$ formic acid before the analysis. The final concentrations of acetylcholine-d4, choline-d9, carnitine-d3, acetylcarnitine-d9, octanoylcarnitine-d3 and palmitoylcarnitine-d3 in each sample was $50 \mathrm{nM}, 1000 \mathrm{nM}$, $50 \mathrm{nM}, 50 \mathrm{nM}, 50 \mathrm{nM}$ and $50 \mathrm{nM}$, respectively.

\subsection{Tandem column FT-MS analysis}

In this study, a NanoLC-2D system (Eksigent, Dublin, CA, USA) coupled to a LTQ-Orbitrap Discovery mass spectrometer (Thermo Fisher Scientific, Bremen, Germany) was used. The two independent binary gradient pumps of our LC arrangement allowed achieving a two-dimensional separation and efficient online solvent mixing without the use of a second HPLC system. A capillary RP column and a HILIC column were coupled in a tandem mode through a nano T-piece. One of the ports of the T-piece was connected to the capillary pump (Channel 1 ) in the Eksigent LC. The inlet line of the RP column was connected to the autosampler, which was coupled with the nanoflow pump (Channel 2) in the Eksigent LC. The outlet line of the HILIC column was coupled with the ESI-MS source. For the primary separation, a C18 column (100 mm $\times 150 \mu \mathrm{m}$ I.D.) packed with $5 \mu \mathrm{m}$ particles (ReproSilPur C18 AQ Dr. Maisch GmbH, Ammerbuch-Entringen, Germany) was used and the secondary separation was performed with an HILIC column $(250 \mathrm{~mm} \times 200 \mu \mathrm{m}$ I.D.) with $5 \mu \mathrm{m}$ particles (Polyhydroxyethyl Aspartamide, PolyLC, Columbia, MD, USA). The RPLC mobile phase was composed of $0.2 \%$ formic acid in water $(A)$ and acetonitrile (B) using the following gradient program at a flow rate of $0.4 \mu \mathrm{L} / \mathrm{min}$ : $0-10 \mathrm{~min}$, linear gradient $2-30 \%$ (B); $10-25 \mathrm{~min}$, linear gradient $30-70 \%$ (B); 25-35 min, linear gradient $70-90 \%$ (B); 35-40 min, returning linear gradient 90-2\% (B); 40-45 min, 
isocratic $2 \%$ (B). The HILIC mobile phase composition was $1 \%$ formic acid, $50 \mathrm{mM}$ ammonium hydroxide in water $(\mathrm{A})$ and $0.2 \%$ formic acid, $10 \mathrm{mM}$ ammonium hydroxide in $95 \%$ acetonitrile (B). To the RPLC mobile phase run-out flow of $0.4 \mu \mathrm{L} / \mathrm{min}$, the following HILIC gradient at a flow rate of $3 \mu \mathrm{L} / \mathrm{min}$ was added via a T-piece: 0-3 min, isocratic 99\% (B); 3-12 min, linear gradient 99-60\% (B); $12-25 \mathrm{~min}$, isocratic $60 \%$ (B); $25-40 \mathrm{~min}$, returning linear gradient $60-99 \%$ (B); 40-45 min, isocratic 99\% (B). A pre-equilibration period of 10 min was used between each run and the injection volume was $1 \mu \mathrm{L}$. The LTQ-Orbitrap mass spectrometer was equipped with a nano-electrospray ion source operating in positive ion mode. For the nanospray ionization, the two-column system was connected to a tip emitter (stainless steel, O.D. $150 \mu \mathrm{m}$, I.D. $30 \mu \mathrm{m}$, Thermo Fisher Scientific). The ion spray voltage was $1.8 \mathrm{kV}$, the capillary temperature was set at $300^{\circ} \mathrm{C}$ and the capillary voltage was $31 \mathrm{~V}$. To increase the sensitivity of the mass analysis, the acquisition was performed in selected ion monitoring (SIM) mode, which was a data acquisition mode conducted in the ion trap for ion filtration and accumulation. For each analyte investigated, a SIM scan event was used in which the scan range was set as $\mathrm{M}^{+} \pm 1 \mathrm{~m} / \mathrm{z}$

The mass spectrometer was calibrated using the manufacturer's calibration standard mixture. The SIM mass spectra were acquired in profile mode with a setting of 30,000 resolution (FWHM) at $\mathrm{m} / \mathrm{z}$ 400. The entire LC/MS system and data processing were performed using Xcalibur software (Thermo Fisher Scientific). Each QAC peak area was integrated using the extraction ion chromatogram (EIC) technique in which each chromatogram is defined by its characteristic mass peak within $10 \mathrm{ppm}$ mass tolerance. The QACs were relative quantified by calculating the peak area ratio of a QAC to its corresponding internal standard. Choline, acetylcholine, carnitine, acetylcarnitine, octanoylcarnitine and palmitoylcarnitine were quantified using their own stable isotope-labeled internal standards. Propionylcarnitine was quantified using the acetylcarnitine internal standard; hexanoyl-, decanoyl- and lauroylcarnitine with the octanoylcarnitine internal standard and myristoylcarnitine with the palmitoylcarnitine internal standard. Each extract from different brain areas of three tree shrews was analyzed for four times. The peak area ratio between an analyte and its corresponding internal standard was then averaged for comparative analysis $(n=12)$.

\section{Results and discussion}

\subsection{Sample preparation}

Sample preparation has a critical influence on the detection capability and reproducibility of the analytical method. In the present study, the animal brains were immediately stabilized using microwave irradiation to prevent the degradation of QACs in the post-mortem period. In order to extract the analytes from brain tissue with low content of salts and proteins, the sample preparation was conducted using $100 \%$ acetonitrile with $0.3 \%$ formic acid [22] but without any drying treatment.

A large number of studies have showed that, in post-mortem period, enzymes can rapidly cause the degeneration of some tissue components such as proteins and peptides in the brain $[23,24]$. The degeneration alters the levels of these endogenous compounds and leads to false estimates of their actual amounts. In the brain, acetylcholine can be rapidly decayed to choline under the hydrolysis of acetylcholinesterase [2]. Similar mechanism exists for acylcarnitines [1]. Tissue stabilization using microwave heating or related techniques is commonplace in proteomics studies, but has not been widely applied for stabilization of neurotransmitter levels. The application of microwave irradiation proved to rapidly terminate the enzyme activity and thus maintain the levels of endogenous components in the brain tissue [21].

Currently, various extraction methods are used for the extraction of acetylcholine, choline and/or carnitines. These methods include solid phase extraction [17], liquid-liquid extraction [18,25], or acidified solvent extraction [22]. Liu and Pasquali [22] described an extraction method that used acidified acetonitrile followed by drying treatment. This method allowed acylcarnitines to be well extracted with low level of interferences from salts and proteins, because of the use of high content of organic solvents [26,27]. In our study, we found that the use of a drying treatment did not allow us to detect several long-chained, hydrophobic carnitines such as myristoylcarnitine and palmitoylcarnitine. They may be irreversibly absorbed by the matrix or other materials such as the filter or vials during the drying treatment and the subsequent reconstitution processing, resulting in highly variable and unreliable estimates specifically for these compounds. In contrast, our extraction method, which did not use a drying step, allowed myristoylcarnitine and palmitoylcarnitine to be extracted along with other QACs, which in turn allowed them to be readily detected by using our newly developed LC-MS method (discussed in the following sections).

\subsection{RP-HILIC-FT-MS analysis}

To acquire the separation of all the targeted QACs in a single HPLC-MS run, we developed a tandem capillary column coupling system. In this setup, the RP column acted as the first column to separate QACs of low polarity such as palmitoylcarnitine, while the HILIC column worked as the second column to separate QACs of high polarity, including acetylcholine, choline, acetylcarnitine, and carnitine. As the system used direct coupling between the two columns, the adjustment of the flow rate and the mobile phase composition was thus required to keep the system working appropriately. To retain the polar compounds in HILIC, the content of acetonitrile in the eluate from the RP column was enhanced by online mixing with high content of acetonitrile solvent. As the HILIC column is coupled post the RP column, the pre-column pressure of the RP column will be inevitably high due to the cumulative effect of the backpressure on the two columns. To make the system works at the appropriate pressure range, the RP column had a smaller diameter and shorter length than the HILIC column. In the experiments, the mobile phase for the RP column was delivered at $0.4 \mu \mathrm{L} / \mathrm{min}$ by using the nanoflow pump of the two dimension LC, while the online mixing solution was delivered at $3 \mu \mathrm{L} / \mathrm{min}$ by using a capillary pump. After online mixing, the calculated content of acetonitrile in the initial mobile phase on the HILIC column was around $83 \%$, which was high enough for maintaining the HILIC separation mechanism. The profiles of the mobile phase gradient were controlled by a single software. This setup enabled to separate and detect all the QACs of interest from the brain extracts (see Fig. 2 the LC-MS analysis of brain extract). In the experiments, all the analytes were measured by high resolution mass spectrometry and identified within $10 \mathrm{ppm}$ mass tolerance and with their characteristic retention time compared to their standards $[28,29]$.

It remains a challenging task to develop a fast and reliable analytical method that allows the simultaneous determination of groups of compounds with different polarities, e.g., QACs targeted in the present study. Ion-pairing chromatography methods have been described for the analysis of the acylcarnitines as well as for acetylcholine and choline [18,19]. However, the use of buffers and ion-pair reagents commonly requires long condition time for columns when using gradient elution profile. It could also suppress the ESI process and diminish the signal intensity, which may demand complex sample preparation steps such enrichment of QACs. Recent studies showed that RP-LC-MS could be directly 

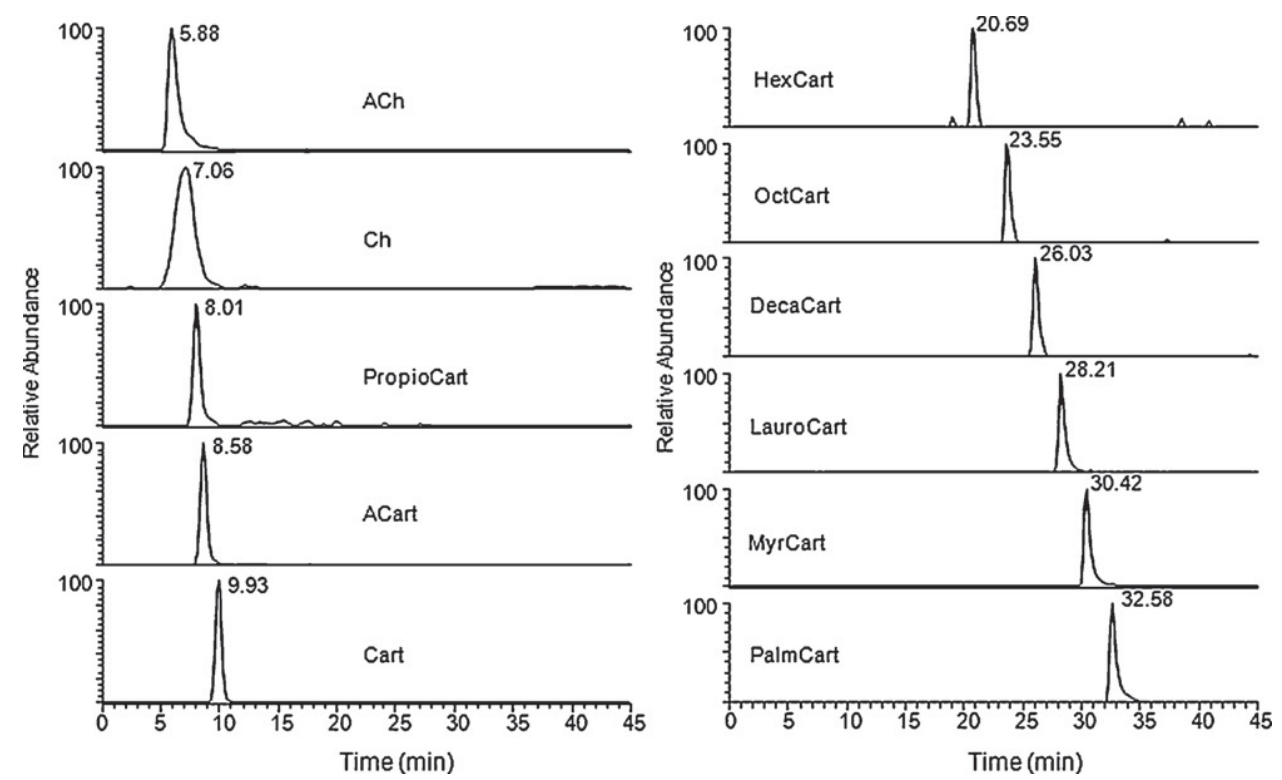

Fig. 2. Representative chromatograms of a striatum extract. The capillary RP-HILIC-FT-MS setup allows the separation and detection of all the targeted QACs in striatum. The RP column provides separation for hydrophobic QACs including hexanoylcarnitine, octanoylcarnitine, decanoylcarnitine, lauroylcarnitine, myristoylcarnitine and palmitoylcarnitine. The HILIC column provides separation for acetylcholine, choline, carnitine, acetylcarnitine and propionylcarnitine. The striatum sample is prepared using $16 \mu \mathrm{L}$ solution per $1 \mathrm{mg}$ of tissue. Each QAC was detected with signal to noise ratio $\gg 10$.

used for analysis of acylcarnitines but not free carnitine, acetylcholine or choline [30], while HILIC-MS allowed polar QACs such as acetylcholine and choline to be separated and detected $[13,14]$. Although a recent study has shown that acetylcarnitine and palmitoylcarnitine could be analyzed using HILIC-MS [31], the elution times of the two QACs were short and very close, reflecting the low separation and peak capability of HILIC-MS for a large number of QACs, in particular for hydrophobic ones. In contrast, our results showed that the direct coupling between RP and HILIC columns allowed good separation of multiple QACs, despite large differences in hydrophobicity. Compared to other 2D-LC setups [32-36], our system was relatively simple and easy to operate. The system did not use an extra valve between the two columns, and all the flow rates were controlled by a single 2D nanoLC system. The system also retains the advantage of HILIC for highly sensitive detection, because the high organic mobile phase content used in HILIC facilitates the desolvation and compound ionization in the electrospray process. In addition, the use of capillary columns could also increase the sensitivity in the detection of QAs compared to standard columns, because of their small diameters.

In our study, the nine carnitines, together with acetylcholine and choline were selected regarding their biological relevance during cholinergic transmission and related energy metabolism. They together represented compounds of a large range of hydrophobicities. Our developed RP-HILIC-FT-MS method showed good peak capacity for separation of not only polar carnitines, but also hydrophobic carnitines. In the brain exist various carnitines with different chain lengths. As most other carnitines have median or long acyl chains and consequently have higher hydrophobicities than the free carnitine, these carnitines could thus be also retained and separated, if necessary, on the first C18 column of our system.

\subsection{Analysis of tree shrew brain extracts}

Having achieved the separation of the targeted analytes on the tandem column system, the method was further tested to evaluate its suitability for a relative determination of the targeted QACs in three different brain regions (striatum, prefrontal cortex and hippocampus). Each QAC was detected in selected ion monitoring (SIM) mode and the peak area was integrated using the extraction ion chromatogram (EIC) technique with $10 \mathrm{ppm}$ mass tolerance. The analytes were relative quantified by measuring the peak area ratio of a QAC to its corresponding internal standard.

The stability of a two dimension LC-MS is very important for continuous quantitative analysis of large set of biological samples. Our capillary RP-HILIC-MS system allowed continuous analysis of the brain tissue extracts for a period lasting up to two weeks. The high stability could be mainly attributed to the sample preparation procedure. In complex biological samples, some substances do not dissolve well in high content of organic solvents and therefore have to be removed prior to HILIC analysis. Our initial tests showed that the brain tissue extracts obtained using low content of acetonitrile could often result in the blockade of the HILIC column. In contrast, our current sample treatment was able to efficiently avoid this issue because brain tissues were extracted with high content of acetonitrile and diluted with high content of water. In this way, the substances that are poorly soluble in high content acetonitrile or water were removed in the sample preparation step.

The SIM analysis conducted on FT-MS allowed each QAC detected with high intensity and high stability. The present study targeted developing a relative, rather than an absolute, quantitative method for differential analysis of multiple QACs in the brain. Thus some analytical characteristics, such as the recovery and LOD, were not determined as required in an absolute quantitative analysis. Although LOD was not measured in the present study, we checked the MS signal to noise ratio of each analyte and found all of them were much higher than 10 in the brain samples, a high value allowing differential analysis. Similar to relative quantitative analysis studies commonly conducted in metabolomics and proteomics for endogenous substances [37-39], we monitored the CVs of analyte peak area/IS peak area ratios for quality control [40]. Using three technical replicates prepared from homogenized tissues (see Section 2), we were able to demonstrate the high precision of our method as shown by coefficients of variation (CVs) among measurements of under $17 \%$ for all the QACs (see Fig. 3).

We further examined the regional distribution of the QACs within the three brain regions. Interestingly, we observed pronounced differences in the levels of some QACs across the different 


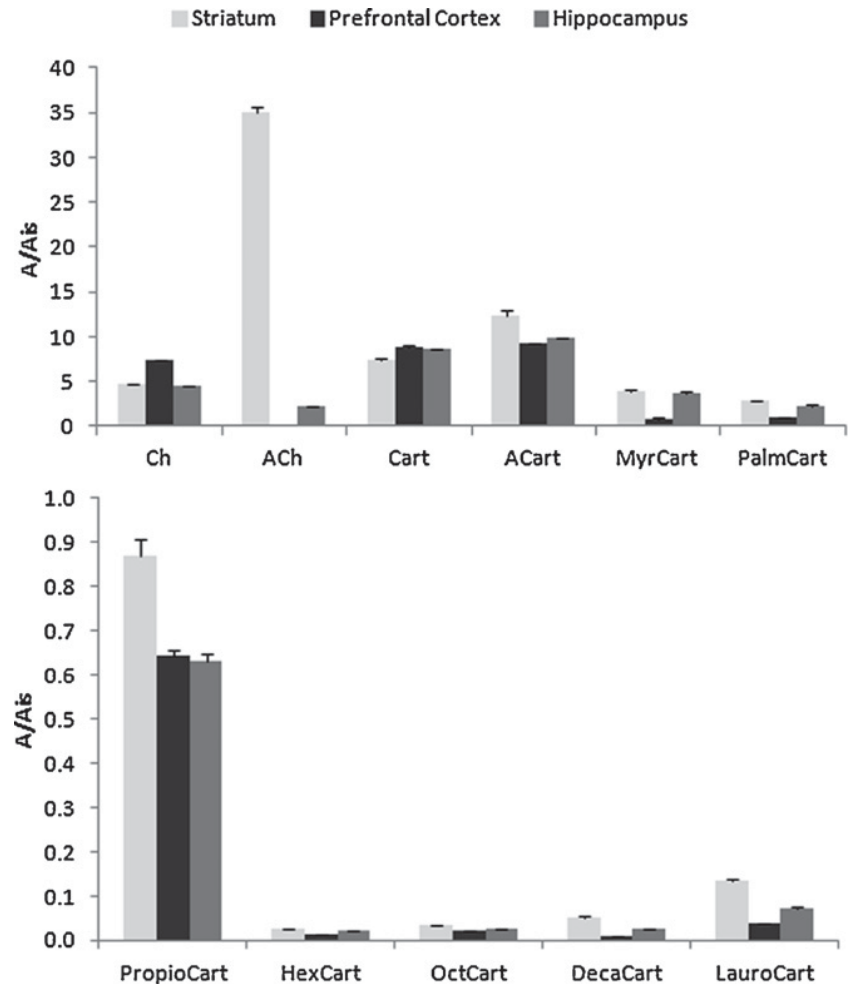

Fig. 3. Average relative amounts of the QACs (analyte peak area/IS peak area) in striatum, prefrontal cortex and hippocampus of three tree shrew brains. The values were expressed as mean \pm s.e.m. The final concentration of internal standard choline d9 in each sample was $1 \mu \mathrm{M}$. The concentrations of other internal standards were $50 \mathrm{nM}$.

brain areas (see Fig. 3). The highest content of acetylcholine was found in the striatum followed by hippocampus and prefrontal cortex. These results are consistent with previous findings in rat and mouse brain, obtained using pyrolytic gas chromatographic methods $[41,42]$. Our findings are also consistent with the known anatomical distribution of cholinergic neurons, as the striatum contains many cholinergic interneurons and the hippocampus is a major projection target of cholinergic cells originating in the medial septum [43].

We also found differences in the distribution of the acylcarnitines in the three brain regions. While carnitine and the short-chain acetylcarnitine and propionylcarnitine exhibited relatively little variation in their regional levels, differences were more pronounced for medium- and long-chain acylcarnitines. These QACs showed similar results to acetylcholine, with higher levels in striatum and hippocampus than in prefrontal cortex. Interestingly, the differential expression between brain areas appeared to correlate with QAC chain length, with highest values observed for long-chained compounds including decacarnitine, lauroylcarnitine, myristoylcarnitine and palmitoylcarnitine.

Only few studies have so far addressed the differential distribution of carnitine and its acyl derivatives in specific brain regions $[44,45]$, although these studies have generally not distinguished among different long-chained acylcarnitines. In agreement with our results, these studies have also shown a heterogeneous distribution of long-chain acylcarnitines in the areas investigated, with low content observed in the frontal cortex and high content observed in regions not examined in the present study such as the cerebellum and hypothalamus [44].

Taken together, the results obtained with our method are consistent with the available literature. QACs carry out important functions in brain and central nervous system, contributing for example to neural activity, membrane plasticity, energy metabolism and protein modification. Our method is suited for future studies of QAC involvement in physiological and biochemical pathways related to these processes.

\section{Conclusions}

We have presented an integrated method for the analysis of multiple endogenous QACs. This method allows 11 QACs extracted, separated and detected from homogenized brain extracts. Although these QACs have very different hydrophobicities, their separations are facilitated by using the tandem connection of both reversed phase column and HILIC column. The combinational use of high accuracy MS and tandem capillary columns permits the detection of targeted QACs with high selectivity and sensitivity. Our relative quantitative method allowed profiling QACs in the different brain areas of tree shrews. Due to the functional importance of QACs in the brain and central nervous system, we anticipate the application of our method in basic neuroscience and pharmaceutical research.

\section{Acknowledgments}

This work was supported by the SNF R'Equip 316000-121308 and a EURYI award to GR.

\section{References}

[1] L.L. Jones, D.A. McDonald, P.R. Borum, Prog. Lipid Res. 49 (2010) 61.

[2] M. Sarter, V. Parikh, Nat. Rev. 6 (2005) 48

[3] C. Ranganath, G. Rainer, Nat. Rev. 4 (2003) 193

[4] M. Sarter, V. Parikh, W.M. Howe, Nat. Rev. 10 (2009) 383.

[5] S.H. Zeisel, K.A. da Costa, Nutr. Rev. 67 (2009) 615.

[6] M. Alkondon, E.F. Pereira, W.S. Cortes, A. Maelicke, E.X. Albuquerque, Eur. J. Neurosci. 9 (1997) 2734.

[7] C.J. Rebouche, H. Seim, Annu. Rev. Nutr. 18 (1998) 39

[8] S. Ando, T. Tadenuma, Y. Tanaka, F. Fukui, S. Kobayashi, Y. Ohashi, T. Kawabata, J. Neurosci. Res. 66 (2001) 266

[9] A. Imperato, M.T. Ramacci, L. Angelucci, Neurosci. Lett. 107 (1989) 251.

[10] A. Wawrzenczyk, K.A. Nalecz, M.J. Nalecz, Neurochem. Int. 26 (1995) 635.

[11] H.L. White, P.W. Scates, Neurochem. Res. 15 (1990) 597.

[12] N.B. Cech, C.G. Enke, Mass Spectrom. Rev. 20 (2001) 362.

[13] P. Uutela, R. Reinila, P. Piepponen, R.A. Ketola, R. Kostiainen, Rapid Commun. Mass Spectrom. 19 (2005) 2950.

[14] X. Zhang, A. Rauch, H. Lee, H. Xiao, G. Rainer, N.K. Logothetis, Rapid Commun. Mass Spectrom. 21 (2007) 3621.

[15] M.E. Hows, A.J. Organ, S. Murray, L.A. Dawson, R. Foxton, C. Heidbreder, Z.A. Hughes, L. Lacroix, A.J. Shah, J. Neurosci. Methods 121 (2002) 33.

[16] M.Y. Zhang, Z.A. Hughes, E.H. Kerns, Q. Lin, C.E. Beyer, J. Pharm. Biomed. Anal. 44 (2007) 586

[17] P.E. Minkler, M.S. Stoll, S.T. Ingalls, S. Yang, J. Kerner, C.L. Hoppel, Clin. Chem. 54 (2008) 1451.

[18] L. Vernez, M. Wenk, S. Krahenbuhl, Rapid Commun. Mass Spectrom. 18 (2004) 1233.

[19] Y. Zhu, P.S. Wong, M. Cregor, J.F. Gitzen, L.A. Coury, P.T. Kissinger, Rapid Commun. Mass Spectrom. 14 (2000) 1695.

[20] P.E. Minkler, S.T. Ingalls, C.L. Hoppel, Anal. Chem. 77 (2005) 1448.

[21] F.Y. Che, J. Lim, H. Pan, R. Biswas, L.D. Fricker, Mol. Cell. Proteomics 4 (2005) 1391.

[22] A. Liu, M. Pasquali, J. Chromatogr. B: Analyt. Technol. Biomed. Life Sci. 827 (2005) 193.

[23] M. Fountoulakis, R. Hardmeier, H. Hoger, G. Lubec, Exp. Neurol. 167 (2001) 86.

[24] K. Skold, M. Svensson, M. Norrman, B. Sjogren, P. Svenningsson, P.E. Andren, Proteomics 7 (2007) 4445.

[25] S.H. Kirsch, W. Herrmann, Y. Rabagny, R. Obeid, J. Chromatogr. B: Analyt. Technol. Biomed. Life Sci. 878 (2010) 3338.

[26] C. Polson, P. Sarkar, B. Incledon, V. Raguvaran, R. Grant, J. Chromatogr. B 785 (2003) 263.

[27] K. Claeson, F. Aberg, B. Karlberg, J. Chromatogr. B 740 (2000) 87.

[28] A. Kaufmann, P. Butcher, K. Maden, S. Walker, M. Widmer, Rapid Commun. Mass Spectrom. 25 (2011) 979.

[29] A. Kaufmann, P. Butcher, K. Maden, S. Walker, M. Widmer, Talanta 85 (2011) 991.

[30] A.K. Ghoshal, T. Guo, N. Soukhova, S.J. Soldin, Clin. Chem. 51 (2005) A138.

[31] Z. Liu, A.E. Mutlib, J.Y. Wang, R.E. Talaat, Rapid Commun. Mass Spectrom. 22 (2008) 3434.

[32] M. Gilar, P. Olivova, A.E. Daly, J.C. Gebler, Anal. Chem. 77 (2005) 6426.

[33] M.P.Y. Lam, S.O. Siu, E. Lau, X.L. Mao, H.Z. Sun, P.C.N. Chiu, W.S.B. Yeung, D.M. Cox, I.K. Chu, Anal. Bioanal. Chem. 398 (2010) 791. 
[34] S. Louw, A.S. Pereira, F. Lynen, M. Hanna-Brown, P. Sandra, J. Chromatogr. A 1208 (2008) 90

[35] Y. Wang, R. Lehmann, X. Lu, X.J. Zhao, G.W. Xu, J. Chromatogr. A 1204 (2008) 28.

[36] Y. Wang, X. Lu, G.W. Xu, J. Sep. Sci. 31 (2008) 1564.

[37] L.N. Mueller, M.Y. Brusniak, D.R. Mani, R. Aebersold, J. Proteome Res. 7 (2008) 51.

[38] A. Nilsson, M. Falth, X. Zhang, K. Kultima, K. Skold, P. Svenningsson, P.E. Andren, Mol. Cell. Proteomics 8 (2009) 1094.

[39] A. Koulman, M. Cao, M. Faville, G. Lane, W. Mace, S. Rasmussen, Rapid Commun. Mass Spectrom. 23 (2009) 2253.
[40] E. Tareke, J.F. Bowyer, D.R. Doerge, Rapid Commun. Mass Spectrom. 21 (2007) 3898.

41] M. Fujiwara, Y. Watanabe, Y. Katayama, Y. Shirakabe, Eur. J. Pharmacol. 51 (1978) 299

[42] D.E. Schmidt, Neuropharmacology 15 (1976) 77

[43] A.G. Karczmar, V.P. Whittaker, N. Chauhan, A. Christopoulos, J. Lindstrom, G.J. Siegel, Exploring the Vertebrate Central Cholinergic Nervous System, 1st edition, Springer, US, 2007.

[44] N. Bresolin, L. Freddo, L. Vergani, C. Angelini, Exp. Neurol. 78 (1982) 285.

[45] A.L. Shug, M.J. Schmidt, G.T. Golden, R.G. Fariello, Life Sci. 31 (1982) 2869. 